\title{
Su te stenderà la sua ombra. Iconografia e tipologia biblica in due Annunciazioni di Filippo Lippi
}

\begin{abstract}
Riassunto
Il presente studio è dedicato al significato simbolico dell'ombra e della luce in due opere di Filippo Lippi: L'Annunciazione della Cappella Martelli in San Lorenzo a Firenze e il quadro dello stesso soggetto nella Collezione Frick a New York. Nel rinascimento l'ombra che appare nell'Annunciazione viene spesso associata con la forza procreatrice di Dio. Il fatto che anche Filippo Lippi fa ricorso a questo senso dell'ombra è particolarmente evidente nella sua Annunciazione della collezione Frick. È meno noto che l'ombra nell'arte di Filippo ha un duplice senso. In realtà, il contrasto tra luce e ombra in questi quadri non è il risultato dell'esperimentazione con la luce naturale; neppure si tratta di una divergenza - come alcuni hanno sostenuto - causata dal fatto che le due metà del quadro originariamente erano destinate a essere montate separatamente come ali di un organo o un armadio. Ritengo invece che la divergenza di luce, che divide le dette opere in una parte illuminata e una oscurata, sia un gioco inteso a visualizzare il concetto teologico del rapporto tipologico tra i due Testamenti e la realizzazione delle profezie sulla nascita del Messia.
\end{abstract}

\section{Abstract}

This study is devoted to the symbolic significance of shadow and light in two works by Filippo Lippi: The Annunciation in the Martelli Chapel in the church of San Lorenzo in Florence and the painting of the same subject in the Frick Collection in New York. In the Renaissance the shadow that appears in the Annunciation is often associated with the procreative power of God. The fact that Filippo Lippi makes use of this sense of the shadow is particularly evident in his Frick Annunciation. It is less well known that in the art of Filippo the shadow has a dual meaning. In fact, the contrast between light and shade in these pictures was not a result of experimentation with natural light; nor is it a difference - as some have argued - caused by the fact that the two halves of the picture were originally intended to be installed separately like the wings of an organ or an armadio (closet for ex voti). Instead, I believe that the difference in the way in which the two parts of these paintings is illuminated is a pun intended to emphasize the theological concept of typological relationship between the Testaments and the realization of the prophecies about the birth of the Messiah.

\section{Introduzione}

Nel Vangelo secondo Giovanni Cristo definisce se stesso come luce; "Io sono la luce del mondo; chi segue me, non camminerà nelle tenebre ..."(Gv 8, 12). Per questo, nelle rappresentazioni medievali della Maiestas Domini, Dio appare spesso con un libro con lo scritto, ego sum lux mundi. La stessa idea viene riproposta in tante visualizzazioni dell'Annunciazione, dove il momento del 


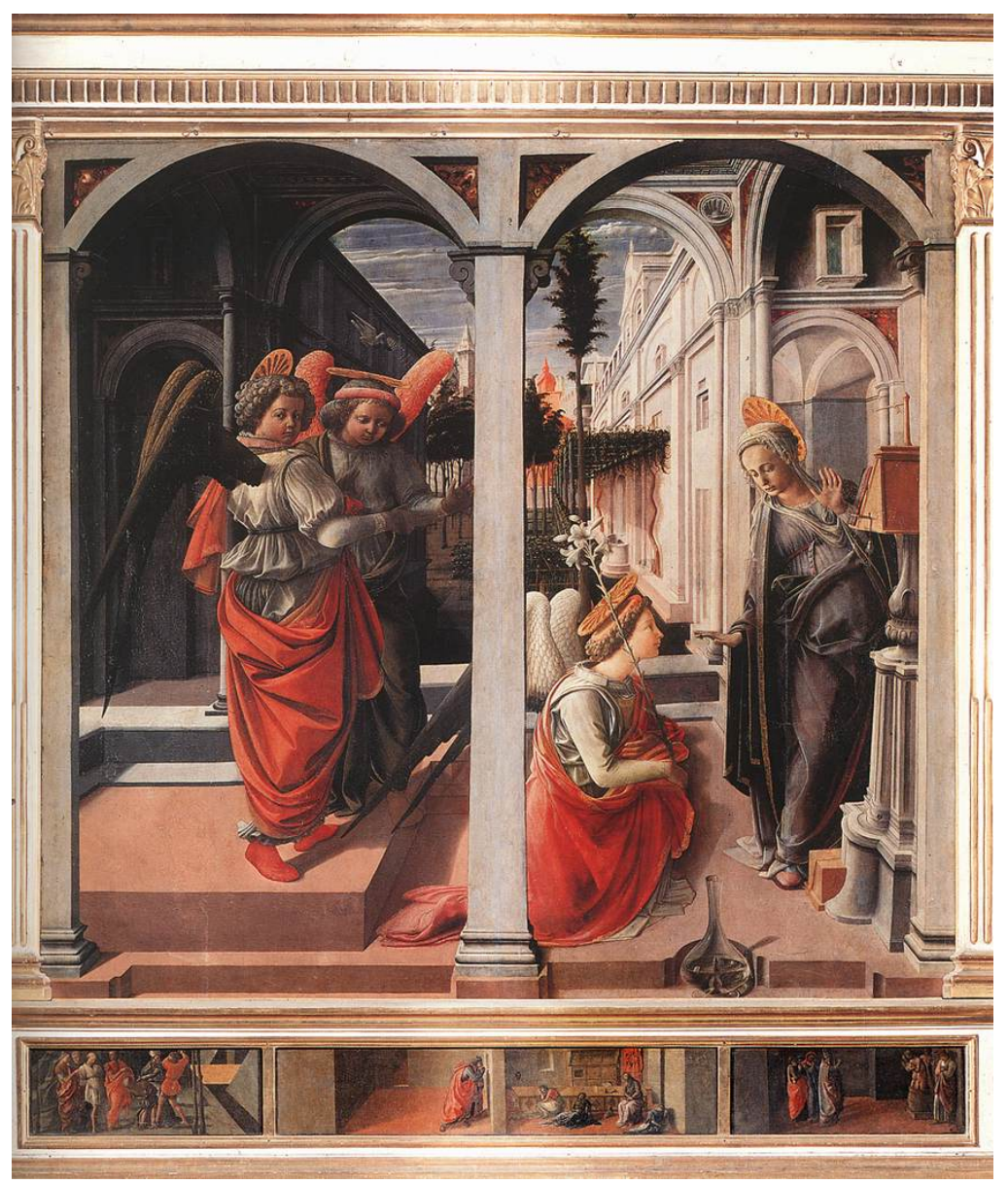

FIG. 1 - Filippo Lippi, Annunciazione, Basilica di S. Lorenzo, Firenze.

concepimento viene indicato con un raggio divino che dall'alto è diretto verso il corpo della Madonna. Ciò che invece è meno noto è che, a volte, l'arte include riferimenti anche alla "forza divina che feconda", che, al contrario di quanto si possa pensare, non è rappresentata dalla luce ma dall'ombra: "Lo Spirito Santo scenderà su di te, su te stenderà la sua ombra la potenza dell'Altissimo" (Lc 1, 35).

Il presente articolo è dedicato allo studio del significato simbolico del rapporto tra luce e ombra nell'arte di Filippo Lippi, con particolare attenzione all'ombra e al suo duplice senso. A tal fine saranno analizzate due rappresentazioni dell'Annunciazione di Filippo Lippi; una sita nella cappella Martelli nella basilica di San Lorenzo a Firenze (FIG. 1), l'altra nella Frick Collection a New York (FIG. 2).

\section{Ricostruzioni}

L'Annunciazione di Filippo a San Lorenzo (1440 ca.) è la prima pala d'altare del quattrocento dove si rinuncia agli elementi gotici quali pinnacoli e cuspidi a favore di uno schema quadrangolare di gusto classico. Secondo Christa Gardner von Teuffel la forma rettangolare dell'opera di Filippo 
FIG. 2 - Filippo Lippi, Annunciazione, Frick Collection, New York.

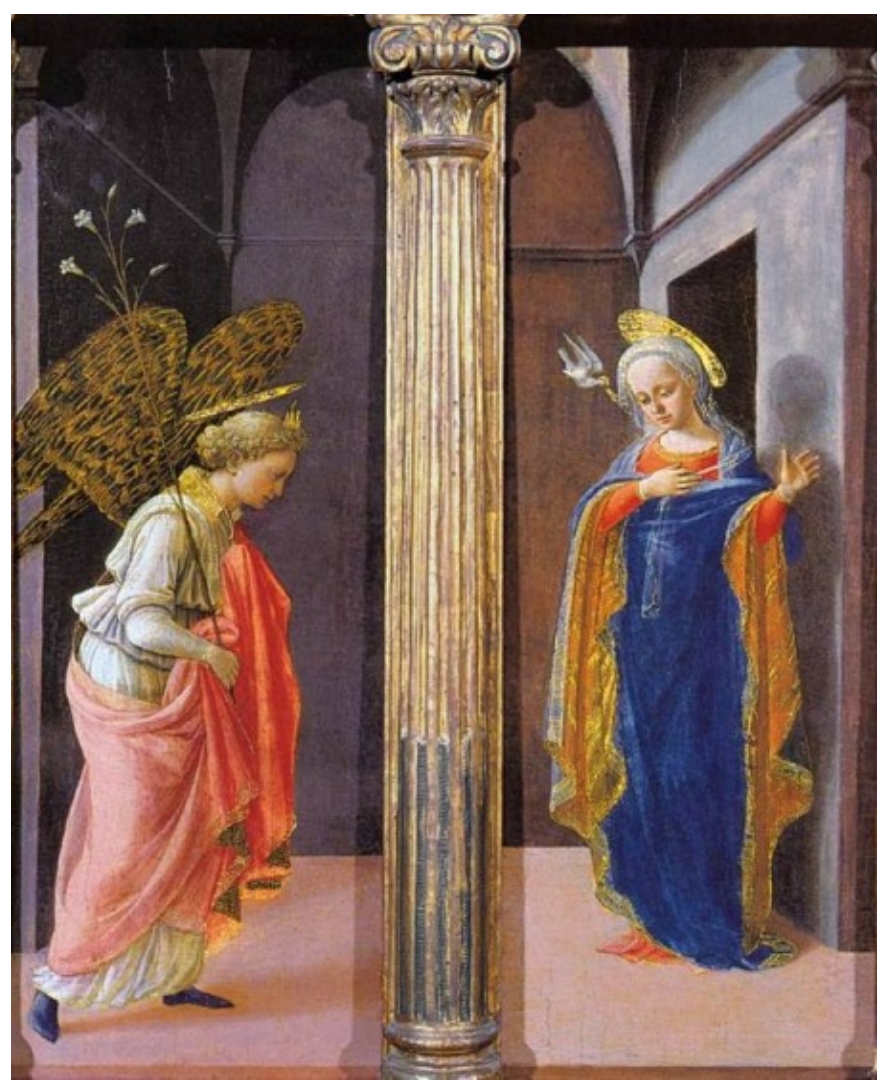

è ispirata dallo stile di Filippo Brunelleschi, architetto responsabile della ristrutturazione della Basilica di San Lorenzo. ${ }^{1}$

Una delle particolarità della pala di Filippo è che la cornice "vera" della scena dell' $A n$ nunciazione circoscrive un'altra cornice, questa volta finta (in quanto dipinta). Quest'ultima è parte integrale dell'architettura dipinta: fa parte della loggia dove si svolge l'azione illustrata nel quadro. Il pilastro finto centrale divide il quadro in due parti uguali, creando così due spazi; in quello a sinistra si vedono due angeli che sono spettatori all'evento principale; in quello a destra le figure centrali, cioè la Vergine e l'angelo Gabriele.

L'opera di Filippo Lippi è carica di significati simbolici, quasi tutti con riferimenti alla verginità di Maria: la caraffa piena di acqua sulla soglia in primo piano, ${ }^{2}$ il giardino nel fondo che probabilmente va inteso come un Hortus conclusus, ecc. ${ }^{3}$ In questo articolo però la nostra attenzione sarà rivolta alla suddivisione in due del quadro per mezzo del pilastro finto, quasi come se fosse un dittico. È una cosa non insolita nell'arte fiorentina. Filippo stesso la ripetè più volte, per esempio nell'Annunciazione che ora si trova nella National Gallery a Washington e, in maniera ancora più prominente, nell'opera dello stesso soggetto ora nella Frick Collection a New York (FIG. 2). Per quanto riguarda l'Annunciazione Frick, non si sa per certo se la cornice attuale rispecchia

1. Gardner von Teuffel 1982, 1-30.

2. Schnitzler 2011,3-15. 
quella originale. Si suppone che le due metà del quadro - quella dell'Angelo a sinistra e quella della Vergine a destra - sono gli scomparti rimanenti di un piccolo altarolo portatile $(64 \times 23 \mathrm{~cm}$ ca.) dove si è persa la parte centrale. Un indizio di questo è che i pannelli costituenti il quadro sono stati tagliati (ma di poco) ai lati, e che sia il livello del pavimento che i dettagli dell'architettura non corrispondono perfettamente tra le due metà. ${ }^{4}$ Ciò non ostante, sembra che la maggior parte degli studiosi, incluso i curatori della Frick, siano d'accordo nell'affermare che la cornice originale dovrebbe anch'essa includere una colonna intercalata tra le parti. ${ }^{5}$

Come per l'Annunciazione Frick, anche per l'Annunciazione di Filippo a S. Lorenzo, si è ipotizzato che le due metà del quadro originariamente non erano direttamente connesse così come oggi le vediamo. Il primo a suggerire una tale ipotesi è stato probabilmente John Shearman, presto seguito da Jeffrey Ruda e Francis Ames-Lewis. Secondo il Ruda è da un documento del 1434 sulla sistemazioni degli altari nella chiesa di San Lorenzo che si può evincere che le due metà del quadro erano destinate a essere montate separatamente, forse come ali di un organo. ${ }^{6}$ Poi si è schierato per la stessa ipotesi sia Eliot W. Rowlands che con tanta sicurezza rivendicava che l'Annunciazione in San Lorenzo certamente era programmata per due pannelli separati, ${ }^{7}$ che Megan Holmes (sia pur con meno convinzione), come riportato nel suo libro su Filippo Lippi: The Carmelite Painter. ${ }^{8}$

Sempre a supporto di questa ipotesi lo studioso Ames-Lewis, in un articolo del 1990, fa riferimento ad alcune osservazioni tecniche che, a suo avviso, svelano una ricomposizione tardiva del quadro. Egli osserva che mentre le tavole laterali sono tenute insieme da morsetti davanti e dietro, tra quelle centrali mancano quelle davanti. Poiché la giunzione tra quest'ultime è da ritenersi meno forte, secondo Ames-Lewis questa è un'indicazione che esse una volta erano separate. ${ }^{9}$

Di parere contrario è invece Christa Gardner von Teuffel che, avendo avuto l'occasione nel 1970 di fotografare il retro dell'opera di Filippo, scoprì che questa parte è composta da tavole non levigate e non preparate, tipiche della tradizionale pala d'altare che permette solamente la visione frontale, differente dalle ali di un organo che devono avere due facce visibili. Dopo un'analisi tecnica accurata ella concluse che le quattro larghe tavole di cui è composto il quadro erano fin dall'inizio unite in modo tale da formare un'unica superficie. ${ }^{10}$

A supporto della sua teoria Gardner von Teuffel evidenzia inoltre che è quanto meno strano posizionare entrambi i protagonisti (l'Arcangelo e la Madonna) nella parte destra e inserire nella parte sinistra due angeli anonimi se poi le due ali sarebbero state destinate ad essere posizionate separatamente. Se invece Gabriele e la Madonna fossero stati posizionati in equilibrio, l'uno di fronte all'altro come nell'Annunciazione Frick, i precursori sarebbero tanti. Basta pensare all'Annunciazione di Giotto nella Cappella degli Scrovegni a Padova, dove l'Angelo e Maria sono posizionati simmetricamente a ciascun lato dell'ingresso al coro. Oppure, più vicino in tempo, il simile posizionamento delle figure nell'affresco di Masolino della Basilica di San Clemente a Roma.

In risposta all'obiezione di Gardner von Teuffel, Ames-Lewis lanciò l'ipotesi che l'Annunciazione di Filippo originariamente era destinata per un'altra posizione rispetto all'attuale.

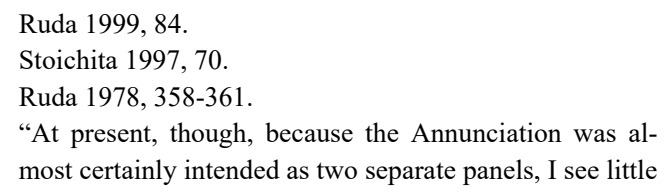

reason to negate entirely Ruda's and Shearman's suggestion." Rowlands 1989, 80, n. 32.

8. Holmes 1999, 125.

9. Ames-Lewis 1990, 155.

10. Gardner von Teuffel 1982, 19. 
Le basi dei pilastri nel quadro di Filippo sono, disse Ames-Lewis, molto simili a quelli usati dal Brunelleschi per la parte nuova della Basilica di San Lorenzo; perciò l'opera era quasi sicuramente destinata per questa parte della chiesa, più precisamente vicino a ciò che si chiamava la Cappella dei Ss. Cosma e Damiano. Da questa posizione l'opera poteva essere ammirata da persone che si trovavano nel transetto, ma, sottolineò - solo la parte con l'angelo Gabriele e la Madonna, non gli angeli anonimi! ${ }^{11}$

Inoltre nella sua tesi Ames-Lewis sostiene che le ante non erano quelle di un organo, bensì di un armadio ex voto. Questa teoria mi sembra alquanto inverosimile e per vari motivi. È una ipotesi basata su un'altra ipotesi; per spiegare come mai le due figure principali non si posizionano simmetricamente, bensì sono stivati nella stessa metà del quadro, Ames-Lewis inventa una differente collocazione originaria (non sufficientemente documentata) da dove solo una metà dell'opera - quella con l'angelo e la Madonna - sarebbe stata visibile al pubblico. Aggiungo anche che questa tesi si basa su indagini tecniche e considerazioni sul contesto fisico e lo spazio circostante il quadro, poco su riflessioni di tipo iconografico. Inoltre, e non di poca importanza, risulterebbe difficile capire il perché le ali di Gabriele e parti delle sue vesti continuino ininterrotti dietro il pilastro centrale nella parte sinistra del quadro.

\section{La colonna di nube}

Date le caratteristiche della pala di Filippo si può capire il perché alcuni studiosi hanno pensato che esse fossero state ideate come parti separate. Personalmente credo però che la spiegazione sia un'altra. Era infatti intenzione del pittore stesso ottenere un effetto che sottolineasse il contrasto tra le due metà. Il pilastro centrale (o la colonna nell'Annunciazione Frick), che divide le due metà, ha di per sé un significato simbolico. Che Filippo conoscesse il simbolismo della colonna lo sappiamo da una terza delle sue opere, l'Annunciazione nella Alte Pinakothek in Monaco di Baviera (FIG. 3). Questo quadro è essenzialmente composto come quello in San Lorenzo: i protagonisti sono posizionati dentro una loggia in primo piano, mentre dietro si vede un giardino. Separato dal resto del giardino da un parapetto si vede un hortus conclusus al centro del quale c'è una colonna circondata da una bassa siepe circolare. Anche se posizionata ad una certa distanza il senso di questa colonna è essenziale; non è un caso che si trova davanti al punto di fuga, cioè il punto verso il quale convergono tutte le linee prospettiche. Considerato che la colonna di per sé non ricopre alcun ruolo architettonico, non può avere altre funzioni se non quella simbolica.

Gli esempi di tale composizione, dove il fulcro dell'attenzione non è né l'Angelo, né la Madonna, ma piuttosto una colonna, sono tante nelle rappresentazioni dell'Annunciazione nell'arte italiana del rinascimento. In un articolo interessante sul simbolismo della colonna nell'arte bizantina e occidentale, Hélène Papastavrou elenca diverse opere di famosi pittori italiani, come Piero della Francesca, Masolino e Antonello da Messina, tutte dove la centralità della colonna è talmente rilevante che non si può non pensare che abbia un significato particolare. ${ }^{12}$ Più diffuso nell'arte occidentale che non in quella orientale, sembra che l'uso simbolico di questo elemento sia ispirato dalla tradizione liturgica bizantina. Nel famoso inno Akathistos (o Acatisto) del VI secolo, dedica- 


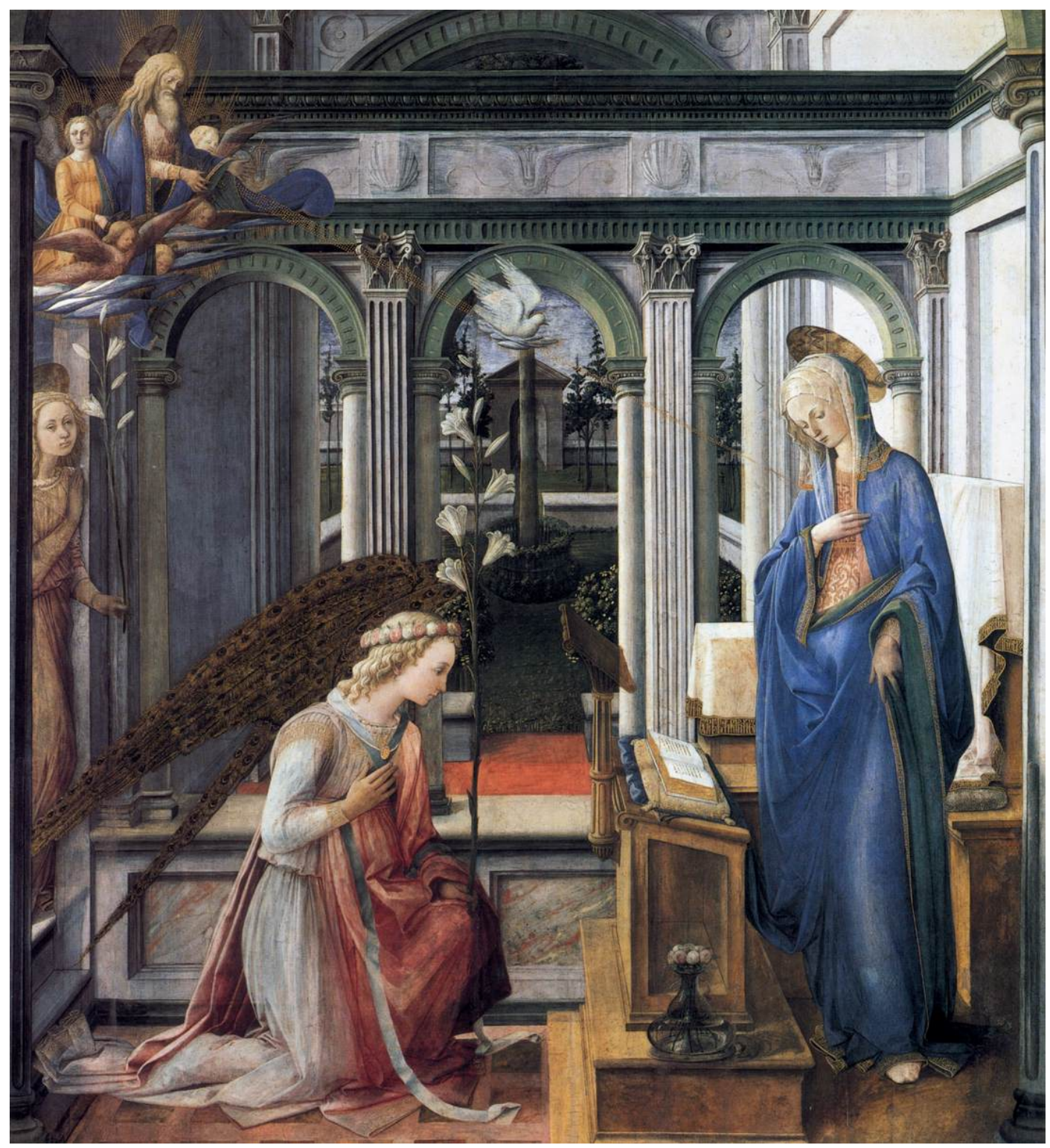

FIG. 3 - Filippo Lippi, Annunciazione, Alte Pinakothek, Monaco.

to alla Theotokos, infatti, la Madonna viene salutata come "colonna della verginità". ${ }^{13}$ In realtà, la denominazione è ancora più antica, perché si trova già nelle omelie di San Giovanni Crisostomo. $^{14}$

La Madonna è una colonna perché è in lei che si rivela l'opera divina, ed è proprio nel momen-

13. Vedi Papastavrou 2002, 26. Spidlik, Guaita, Campa-

14. Spidlik, Guaita, Campatelli 2000, 310. telli 2000,310 . 
to dell'Annunciazione che Dio si rivela come Dio incarnato. La Madonna è una colonna perché la colonna di nube era la forma scelta da Dio quando Egli, nel Vecchio Testamento, si rivelò al popolo d'Israele per condurlo fuori dall'Egitto. Nell'Esodo si legge che "Il Signore marciava alla loro testa di giorno con una colonna di nube, per guidarli sulla via da percorrere, e di notte con una colonna di fuoco per far loro luce, ..." (Esodo 13, 21). La colonna, quindi, è la manifestazione del Dio invisibile che appare davanti al suo popolo solo in maniera indiretta, attraverso impronte. Le sue vestigia ${ }^{15}$ in questo contesto sono luce e ombra; il fuoco durante la notte e la nuvola durante il giorno.

La scelta della nube a forma di colonna che viene menzionato più volte durante l'Esodo come simbolo della verginità e prefigurazione dell'Incarnazione non è casuale. Diversi Padri della Chiesa vedevano nella descrizione dell'Esodo un preannuncio profetico delle parole del Vangelo: "Lo Spirito Santo scenderà su di te, su te stenderà la sua ombra la potenza dell'Altissimo, ..." (Lc 1, 35). ${ }^{16}$ Per questo, forse, nel suo studio sull'Angelico G. Didi-Huberman vede un legame tra l'ombra in Luca e la nube che coprì la tenda del convegno nell'ultimo capitolo dell'Esodo (40, 34-35). ${ }^{17}$

Sappiamo, quindi, che la colonna che appare in alcuni quadri dell'Annunciazione - e sicuramente in casi talmente palesi come quello di Filippo nella Collezione Frick - è segno della presenza divina. Capiamo anche che è proprio nel gettare la sua ombra sulla virginem desponsatam che questa nube, che è Dio, si mostra come forza procreatrice. È per questo che è del tutto naturale chiedersi se la colonna nelle opere di Filippo è di questo tipo, cioè un simbolo della potenza dell'Altissimo che genera per mezzo della propria ombra?

Non sono il primo ad interessarmi all'uso dell'ombra nell'arte del frate carmelitano. Victor I. Stoichita descrive l'ombra creata dal corpo della Madonna che viene proiettata sulla parete a destra, quasi come una specie di fantasma. "Non credo", dice Stoichita, "di sbagliarmi se ritengo che questo quadro rappresenti il momento dell'obumbrabit". ${ }^{18}$ Abbiamo l'esempio di Masaccio che nella Cappella Brancacci racconta la storia di S. Pietro che guarisce gli infermi con la propria ombra. Per questa sua originalità il Masaccio - più vecchio di Filippo solo di pochi anni - viene considerato l'inventore dell'ombra. Circa dieci anni più tardi (1437-1439) Filippo usò l'ombra in una simile maniera - anche qui con significato simbolico - nell'Annunciazione Frick. Un altro esempio più o meno dello stesso periodo è l'Annunciazione del beato Angelico nella cella no. 3 del Convento di San Marco (FIG. 4). ${ }^{19}$ A parte la posizione della Madonna, che nell'Annunciazione del beato Angelico si trova in ginocchio su uno sgabello davanti all'Angelo, i due quadri sono abbastanza simili. In entrambe le opere le figure si trovano in una stanza scarsamente arredata dove si vede la Vergine posizionata molto vicino alla parete destra sulla quale Ella proietta la sagoma ben definita del suo corpo.

Oltre all'ombra della Madonna, qui c'è anche quella del Padre Eterno. Come detto, il Dio invisibile si fa vedere solo attraverso le vestigia che, appunto, può essere l'ombra. Il fatto che il Signore al momento della concezione prende la forma dell'ombra ci fa pensare alla mitologia antica e la storia di Giove e Io. Nelle Metamorfosi Ovidio racconta come Giove, innamorato della sacer-

\footnotetext{
15. Agostino [Santo], De Trinitate, $\mathrm{X}, \mathrm{xi}, 17$ (come citato in

18. Stoichita 1997,70 . Wind 1967, 259-262).

19. Scudieri 2004, 52 .

16. Gambero 1985, 250, n. 34 .

17. Didi-Huberman 1991, 202-205.
} 


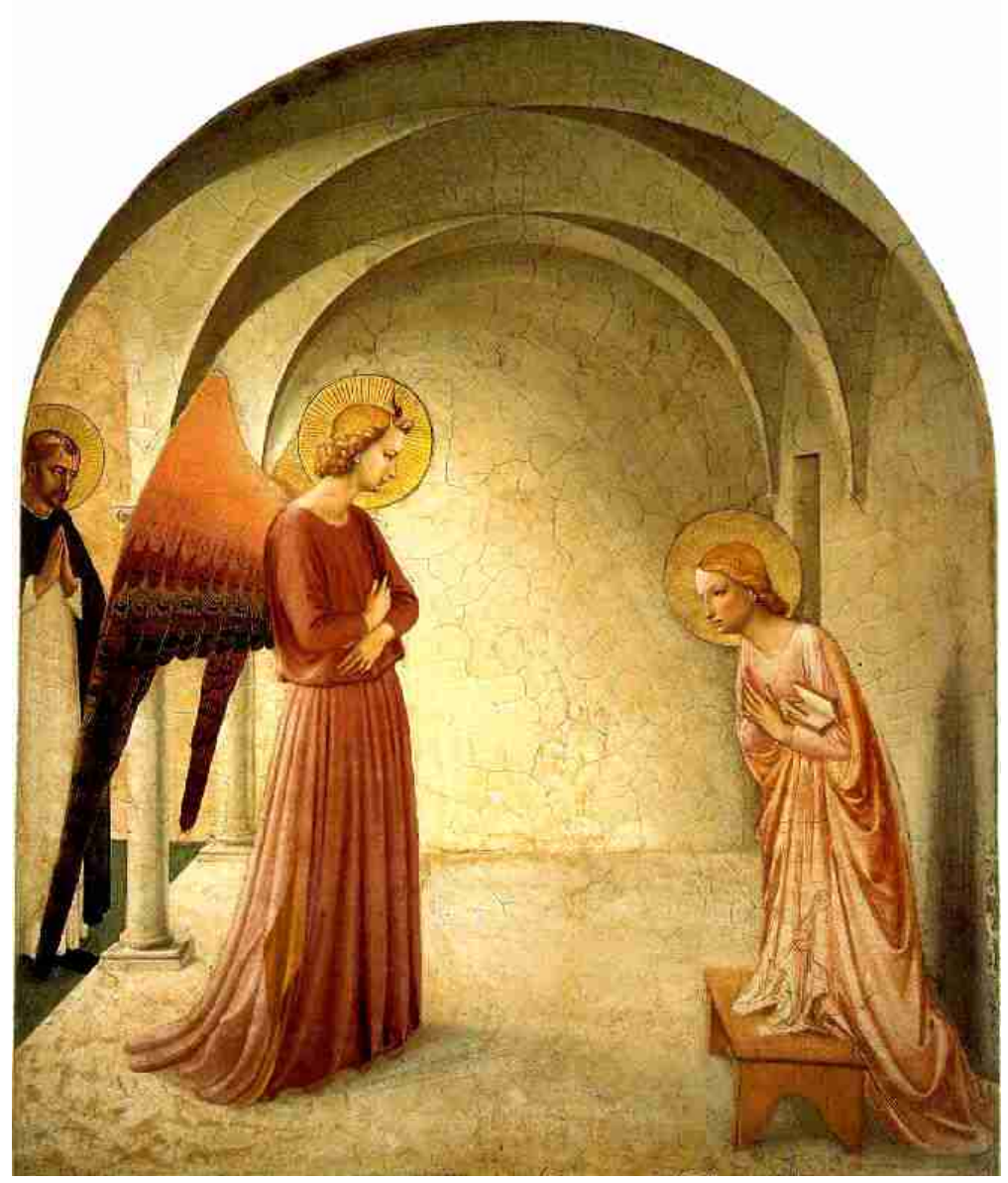

FIG. 4 - Beato Angelico, Annunciazione, Museo di S. Marco, Firenze.

dotessa Io, per sottrarsi alla gelosia della moglie Era, si fece coprire da una fitta nebbia al momento della seduzione della fanciulla.

Il confronto con la mitologia antica non è inopportuno. Nel suo studio sull'Annunciazione di Giotto nella cappella degli Scrovegni, Laurine M. Bongiorno osserva che la luce divina che raggiunge la Madonna in questo caso non è un raggio semplice, ma un fascio di raggi, quasi una pioggia dorata. Già nel secondo secolo San Giustino Martire paragonò la Vergine a Danae. Danae, come si sa, fu rinchiusa in una torre dal padre, il re Acrisio di Argo, che dall'oracolo aveva saputo che sarebbe morto per mano del figlio della figlia. Ciò non ostante, Danae ricevette la visita del dio Zeus, nascosto in una pioggia d'oro, e da questa unione nacque Perseo. ${ }^{20} \mathrm{Il}$ fatto che l'idea rimase viva durante il medioevo è testimoniato dal frate domenicano Franz von Retz, il quale nel Trecento fece lo stesso confronto con la mitologia greca. ${ }^{21}$ 
Peraltro, l'analogia con la tradizione veterotestamentaria è molto vicina, dove infatti si trova la stessa idea dell'acqua che feconda. Nei Salmi si legge che il regno di Dio "Scenderà come pioggia sull'erba, / come acqua che irrora la terra" (Salmo 72, v. 6). ${ }^{22}$ Tale metafora ci ricorda la storia sulla rugiada che si posa sul vello di Gedeone (Giudici 6, 36-40) - un evento spesso riprodotto nei manoscritti tipologici come prefigurazione del concepimento immacolato di Maria.

A mio avviso stiamo di fronte a un gioco di parole su umbra e obumbrabit. La nuvola, come la colonna, segna la presenza del Dio di Abramo, il Dio di Isacco e il Dio di Giacobbe al momento del concepimento di Gesù; al momento cioè che si passa dal dominio del Padre a quello del Figlio, dall'umbra alla veritas. L'ombra che viene gettata dalla colonna di nube è la prefigurazione dell'età della gratia, come se fosse in vigore ciò che funziona bene nella lingua inglese: "to foreshadow", prefigurare. Perciò l'Apostolo nella prima lettera ai Corinzi ammonisce i fratelli che non ignorino "che i nostri padri furono tutti sotto la nuvola, tutti attraversarono il mare, tutti furono battezzati in rapporto a Mosè nella nuvola e nel mare, ..." (1 Cor 10, 1-2).

Era a questo passo tratto dalle lettere di San Paolo che si riferiva Sant'Ambrogio quando parlava della nuvola gentile che oscurò quelli che furono visitati dallo Spirito Santo. Alla fine questa nuvola è arrivata anche sulla Vergine Maria, sulla quale, dice il Santo con riferimento a Luca 1, 35 , si stese l'ombra dell'Altissimo. ${ }^{23}$ Se allora, continua, lo Spirito era in figura, non è forse qui presente in realtà, visto che le Sacre Scritture ci dicono: "Perché la legge fu data per mezzo di Mosè, la grazia e la verità vennero per mezzo di Gesù Cristo" (Gv 1, 17).

\section{Umbra et figura}

San Paolo usa la parola figura come definizione del periodo sub legem (Mosè) che precede quello sotto la "grazia" o la "verità" di Cristo. Etimologicamente la parola figura è quella che abbiamo in prefigurazione e corrisponde al greco typos ( $\tau \dot{\pi} \pi \mathrm{\varsigma})$, che ha dato nome alla "tipologia". Con "tipologia" in questo caso si intende "tipologia biblica", cioè che alcuni episodi raccontati nell'Antico Testamento preannunciano situazioni analoghe nel Nuovo.

"Tipologia" è essenzialmente un concetto teologico, ma considerato che il mondo occidentale è impregnato dal cristianesimo come fattore culturale, è naturale pensare che i suoi principi abbiano avuto un certo influsso sul resto della società, l'arte compresa. Uno che in maniera sistematica ha integrato il pensiero tipologico nelle sue teorie, è il noto filologo tedesco Erich Auerbach (1892-1957). Il binomio Umbra et figura fu adottato da Auerbach in un articolo del 1938 per definire ciò che egli vedeva come il "senso figurale" della letteratura tardomedievale. ${ }^{24}$ L'interpretazione figurale che Auerbach adopera quando esamina la letteratura in vernacolare è legato alla tipologia come concetto teologico che mette in rapporto i due testamenti. Figura, come anche il typos, è un'anticipazione o prefigurazione di un evento futuro che, a sua volta, è più vero e più perfetto del suo precursore. Se l'adempimento delle profezie rappresenta la luce della verità, le profezie stesse non sono altro che ombre di queste realtà.

Dimostrando come la Divina commedia dantesca è strutturata secondo la concezione figurale della storia, Auerbach è stato in grado di rivelare importanti aspetti della mentalità medievale.

\footnotetext{
22. Un'illustrazione di questo passo è riprodotta in Schiller 1971-1972, 43.

23. Ambrogio [Santo] 1995, 315.
} 
Non è sorprendente scoprire che la stessa mentalità si estende all'ambito delle arti figurative. Un esempio di tale uso nella pittura fiorentina scene tratte dal Nuovo Testamento. Il modo in cui l'Angelico e i suoi allievi hanno rappresentato gli episodi dal Vangelo è piuttosto ortodosso; sorprende invece l'inserimento di finti rotoli di pergamena in ogni pannello, due per ogni quadro, uno nella parte superiore, uno lungo il bordo inferiore. Vedendo più da vicino, si scopre che il rotolo superiore porta una frase tratta dal Vecchio Testamento, mentre quello inferiore mostra un versetto dal Nuovo (e ciò vale per l'intera serie dei 35 pannelli). Nella scena dell'Annunciazione, ad esempio, sul cartiglio superiore si legge la prefigurazione Ecce virgo concipiet ... (Is 7, 14), mentre su quello inferiore ci sono le parole Ecce concipies in utero ... (Lc 1, 31) (FIG. 5). L'opera segue il sistema della tipologia biblica dove gli eventi del Vangelo - le azioni e i miracoli di Cristo - vengono considerati come adempimento delle profezie veterotestamentarie. ${ }^{26}$

Ciò che più colpisce è che i finti rotoli che accompagnano ogni riquadro sono illuminati in modo diverso: quello superiore, che contiene il versetto dell'Antico Testamento, è leggermente più scuro di quello inferiore. Così venne ideato un sistema secondo il quale le profezie veterotestamentarie venivano associate all' "ombra", mentre il compimento delle stesse rappresentava la "luce". ${ }^{27}$

La questione resta se Filippo aveva la stessa conoscenza della tipologia biblica di Beato Angelico e se, in tal caso, l'adottò nei suoi quadri. A mio avviso l'aveva, e credo perfino che il binomio umbra et figura è alla base di una particolarità che riguarda la tonalità di colori nelle due Annunciazioni di Filippo che sono alla nostra attenzione qui. Analizzando di nuovo l'Annunciazione di San Lorenzo, vediamo che il giardino di fondo, forse un Hortus conclusus, è limitato a sinistra e a destra da due ali di un grande palazzo. Guardando i gradini in primo piano si deduce che la scena è illuminata da sinistra. Per questo non è strano che l'ala destra del palazzo è illuminata, mentre quella a sinistra resta in buio. La parete di fondo, però, che lega i due opposti, dovrebbe apparire in maniera coerente e uniforme. Non è invece così. ${ }^{28}$ Il problema è l'arcata che separa il primo piano dal giardino, perché, essendo un elemento parallelo alla superficie del quadro, essa dovrebbe essere illuminata alla stessa maniera per tutta la sua lunghezza. Invece, mentre la parte sinistra è scura, quella a destra è molto più chiara; una irregolarità che viene occultata proprio dal pilastro centrale. Infatti, il pilastro centrale divide le due parti in maniera surreale; divide il quadro in due spazi in un modo che non si può comprendere dal punto di vista naturalistico. La parte sinistra, infatti, è molto più scura di quella di destra. Qui le pareti sono dipinte in un color grigio cenere, in netto contrasto con quelle di destra dove specialmente il muro in fondo ha un tono più caldo, tendente verso il rosso arancione.

La colonna, come Filippo la usa, è un trucco ottico che permette un'illuminazione irreale che ha come obiettivo qualcosa che trascende il puro naturalismo: le due metà, anche se teoricamente parti di un unico spazio, sono illuminate come se fossero stanze separate. È indubbio che si tratti di un artificio adoperato da Filippo intenzionalmente con l'obiettivo di comunicare qualcosa per mezzo del gioco di luce e ombra. La parte di sinistra è buia non soltanto perché la luce naturale

\footnotetext{
25. I vari nomi proposti sono Alesso Baldovinetti, Domenico di Michelino, il Maestro della cella 2 di S. Marco, Zanobi Strozzi e Benozzo Gozzoli.

26. Vedi gli studi sulla tipologia dell'Armadio dell'Angelico in Cornell (1925) e Gilbert (2005).
}

27. Vedi il precedente studio sul tema della tipologia nell'arte dell'Angelico in Hodne 2011.

28. Questa differenza viene anche notata dal Ruda, che dice che "... the original paint fields on either side do not quite match in tone.” Ruda 1978, 358-361. 


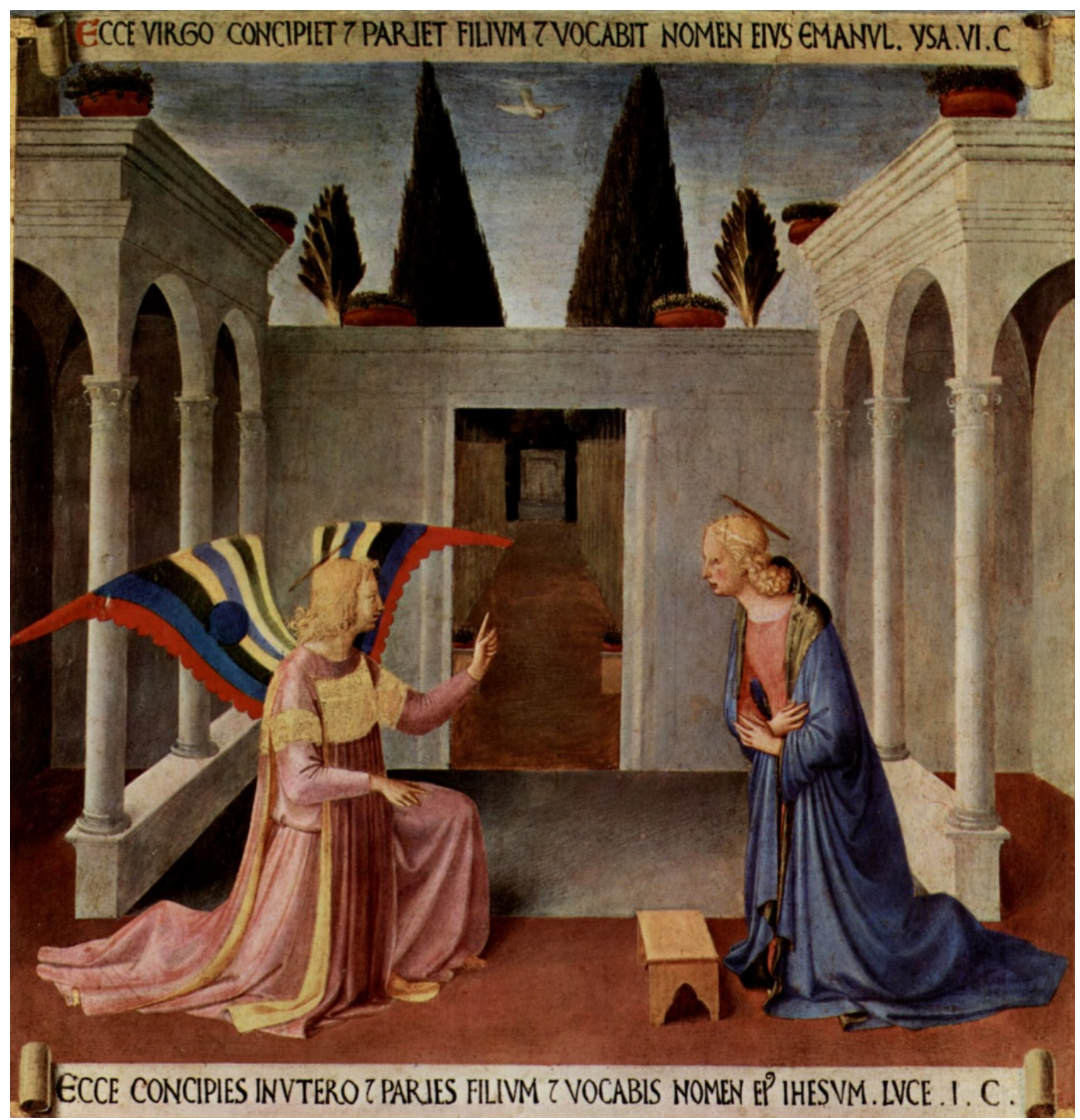

Fig. 5 - Beato Angelico, Annunciazione dall'Armadio degli argenti.

cade da sinistra, ma perché appartiene al tempo incompiuto, alle profezie, alla figura. Di nuovo, lo scuro non è solo ombra, ma umbra nel senso tipologico.

Anche il pavimento è diverso nelle due parti, solo che qui la parte più scura è la destra. Il contrasto tra le due metà potrebbe essere usato come argomento a supporto della teoria che sostiene 
che l'opera originariamente fosse stata ideata come due ali di un organo o un armadio ex voto; oppure potrebbe essere il risultato di un semplice errore da parte del pittore (o perfino di un restauratore). Lo stesso discorso vale anche per l'Annunciazione Frick; anche qui la parte sinistra quella che forse rappresenta il passato - è visibilmente più scura di quella di destra. Secondo me, l'unica spiegazione di questa peculiarità è che Filippo, come l'Angelico, ha scelto l'Annunciazione come soggetto adatto per illustrare l'idea che i termini figura e umbra in qualche modo rappresenta un'anticipazione o una prefigurazione.

Com'è naturale, la narrativa dell'Angelico apre con l'Annunciazione, il primo episodio nella vita di Cristo. A parte l'Armadio degli argenti, l'Angelico dipinse almeno tre Annunciazioni con un tema tipologico di fondo: Nelle pale d'altare a Cortona, a Valdarno e (ora) a Prado le figure principali della Madonna e dell'Angelo sono accompagnati da una scena secondaria che rappresenta la Cacciata dal Paradiso. L'idea fondamentale è di illustrare l'argomento tipologico su come il Peccato originale che il genere umano contrasse per colpa di Eva verrà risanato per mezzo della Madre di Dio.

Sembra che l'Annunciazione come soggetto artistico, almeno a Firenze, in questo periodo era essenziale dal punto di vista tipologico. Perché? In un ciclo di quadri o affreschi che raffigura episodi della vita della Vergine o di Cristo, il punto naturale da cui cominciare è proprio l'Annunciazione. L'Annunciazione segna, per così dire, il passaggio tra il Vecchio testamento e il Nuovo, tra luce e ombra; è il cardine tra due mondi. Come passaggio e legame l'Annunciazione è centrale in doppio senso. In senso teologico è l'illustrazione di uno dei dogmi centrali del cristianesimo, l'Incarnazione. Secondo Bonaventura tutta la storia della Salvezza è orientata e centrata intorno all'Incarnazione che, nel quarto libro delle sue Sentenze, viene definito come plenitudo temporis. ${ }^{29}$ L'Adventus del Figlio di Dio viene qualificato con il termine "pienezza" non perché il suo arrivo determina la fine della storia, ma perché rappresenta la rivelazione delle profezie che prima non si potevano comprendere.

\section{Conclusione}

Lo studio dell'illuminazione e il contrasto tra luce e tenebre completano l'impressione ricevuta da due altri elementi importanti nelle opere di Filippo: il modo in cui il maestro fiorentino attribuì un significato simbolico all'ombra proiettata dal corpo della Madonna e l'inserimento della colonnanube al centro dei quadri. Siamo, quindi, arrivati a significati diversi ma collegati riguardanti la questione dell'"ombra". Filippo Lippi adotta tutte e due nelle sue opere. Prima il gioco di parole su "ombra" e obumbrabit che determina la presenza divina e il modo di concepimento. Secondo, il passaggio da umbra a veritas (oppure lux) che ci riporta al discorso sulle metafore studiate dall'Auerbach. Una "normale" (occidentale) lettura del quadro da sinistra verso destra suggerisce non solo un passaggio tra due fasi dove l'una è segnalata come ombra e prefigurazione dell'altra. Segna uno sviluppo positivo che punta verso un futuro migliore, tutto in conformità con le parole dell'Apostolo che riteneva che la legge di Mosè fosse "solo un'ombra dei beni futuri e non la realtà stessa delle cose, ...” (Ebrei 10,1).

29. Delio 1997, 160. 
A mio avviso è questa la spiegazione della strana composizione delle due Annunciazioni di Filippo Lippi che abbiamo analizzato. La differenza di tono e colore, e la posizione della colonna (oppure pilastro) non rispecchiano uno stato primordiale dove le due metà erano ante di un organo o un armadio, montate a una certa distanza l'una dall'altra. Filippo ha usato questi elementi intenzionalmente per sottolineare un argomento tipologico, spesso ricordato nelle rappresentazioni dell'Annunciazione nel quattrocento fiorentino. Le opere dell'Angelico, come abbiamo visto, richiamano la stessa idea.

Lasse Hodne

Norwegian University of Science and Technology

Department of Art and Media Studies

Trondheim, Norway

Lasse.hodne@ntnu.no 


\section{BIBLIOGRAFIA}

Ambrogio [Santo] 1995: The Book of Saint Ambrose, Bishop of Milan, Concerning the Mysteries, in P. Schaff, Nicene and Post-Nicene Fathers: Second Series, X, Ambrose. Peabody, Mass.

Ames-Lewis, F. 1990: "Fra Filippo Lippi’s S. Lorenzo Annunciation", Storia dell'arte, 69, 255-73.

Auerbach, E. 1938: "Figura", Archivium Romanicum. Nuova rivista di filologia romanza, Firenze.

Bongiorno, L.M. 1958: "The Theme of the Old and the New Law in the Arena Chapel", ArtB 50, No. 1 (Mar., 1968), 11-20.

Cornell, H. 1925: Biblia pauperum, Stoccolma.

Delio, I. 1997: "From Prophecy to Mysticism: Bonaventure's Eschatology in Light of Joachim of Fiore", Traditio, 52, 153-177.

Didi-Huberman, G. 1991: Beato Angelico. Figure del dissimile, Milano.

Gambero, L. 1985: "L'Omelia Sull'ipapante di Anastasio I di Antiochia", Marian Library Studies, 17, no. 19, 236-252.

Gardner von Teuffel, C. 1982: "Lorenzo Monaco, Filippo Lippi und Filippo Brunelleschi: die Erfindung der Renaissancepala", Zeitschrift für Kunstgeschichte XLV/1.

Gilbert, C. 2005: Lex Amoris. La legge dell'amore nell'interpretazione di Fra Angelico, Firenze.

Hodne, L. 2011: "Reading and Viewing Words in Fra Angelico's Typological Paintings", ActaAArtHist, 24 (n.s. 10), 243-263
Holmes, M. 1999: Filippo Lippi: The Carmelite Painter, New Haven, Conn.

Papastavrou, H. 2002: "Le symbolisme de la colonne dans l'art byzantin et occidental", Thesaurismata

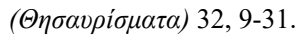

Reau, L. 1956-1957: Iconographie de l'art chrétienne, II, Paris.

Rowlands, E.W. "Filippo Lippi and His Experience of Painting in the Veneto Region", Artibus et Historiae, 10, No. 19 (1989), 53-83.

Ruda, J. 1978: “A 1434 Building Programme for San Lorenzo in Florence", The Burlington Magazine, 120, No. 903.

Ruda, J. 1999: Fra Filippo Lippi: Life and work, London.

Schiller, G. 1971-1972: Iconography of Christian art, I, Greenwich Conn.

Schnitzler, N. 2011: "The Beam of Grace and the Ocular Paradigm. Some Remarks on the Relation Between Late Medieval Theology and Art", in Angels, Devils. The Supernatural and its Visual Representation (a cura di G. Jaritz), Budapest \& New York.

Scudieri, M. 2004: Gli affreschi dell'Angelico a San Marco. Firenze-Milano.

Spidlik, T., Guaita, G. Campatelli, M. (a cura di) 2000: Testi mariani del secondo millennio, Roma.

Stoichita, V. I. 1997: Short History of the Shadow, London.

Wind, E. 1967: Pagan Mysteries in the Renaissance, Harmondsworth. 